\title{
Laryngeal Paraganglioma with Chronic Cough: A Case Report
}

\author{
Peter Papagiannopoulos ${ }^{1}\left(\mathbb{D}\right.$, Anish Raman ${ }^{2}$ (D), Courtney Miller ${ }^{2}$, Miral Jhaveri $^{3}$, Ritu Ghai ${ }^{4}$, \\ Inna Husain ${ }^{1}$ \\ ${ }^{1}$ Department of Otorhinolaryngology - Head and Neck Surgery, Rush University Medical Center, Illinois, USA \\ ${ }^{2}$ Rush University Medical Center, Rush Medical College, Illinois, USA \\ Case Report ${ }^{3}$ Department of Radiology, Rush University Medical Center, Illinois, USA \\ ${ }^{4}$ Department of Pathology, Rush University Medical Center, Illinois, USA
}

Abstract

ORCID IDs of the authors: P.P. 0000-0002-4209-9980; A.R. 0000-0002-1726-9685;

Cite this article as: Papagiannopoulos $P$, Raman A, Miller C, Jhaveri M, Ghai R, Husain I. Laryngeal Paraganglioma with Chronic Cough: A Case Report. Turk Arch Otorhinolaryngol 2018; 56(4):233-6.

This study was presented at the AAO-HNSF Annual Meeting \& OTO Experience, October 7-10, 2017, Illinois, USA.

Corresponding Author:

Anish Raman; anish_raman@rush.edu

Received Date: 21.06 .2018

Accepted Date: 27.08 .2018

Available Online Date: 21.12.2018

(c) Copyright 2018 by Official Journal of the Turkish Society of Otorhinolaryngology and Head and Neck Surgery Available online at www.turkarchotolaryngol.net DOI: 10.5152/ta0.2018.3625
Laryngeal paragangliomas are rare lesions originating from paraganglion cells within the supraglottis or subglottis. As per the latest review, only 76 such cases have been reported in the literature. Symptoms typically include dysphonia or dysphagia. We present, to the best of our knowledge, the first known case of laryngeal paraganglioma with chronic cough as the primary complaint. A 77-year-old male presented with chronic cough. Flexible laryngoscopy revealed a supraglottic submucosal mass emanating from the region of the right false vocal cord and aryepiglottic fold. Postcontrast computed tomography scan showed a well-defined intensely enhancing mass arising from the right paraglottic space and bulging into the right pyriform sinus. Biopsies and immunohistochemical markers supported the diagnosis of paraganglioma. A complete submucosal excision of the mass via a right transcervical approach with tracheostomy was performed. Postoperatively, the patient's cough resolved. Laryngeal paragangliomas are rare tumors that are known to present with dysphonia or dysphagia. This is the first case report of a patient presenting with chronic cough as the primary complaint.

Keywords: Larynx, paraganglioma, cough, diagnosis, surgery

\section{Introduction}

Laryngeal paragangliomas are an exceedingly rare subset of neuroendocrine tumors that arise from the paraganglion cells of the supraglottis or subglottis. As per the latest review, only 76 such cases have been reported in the literature (1).

Neuroendocrine tumors can be classified into two groups based on their origin: epithelial or neural crest-derived $(1,2)$. This distinction is highly relevant because the prognosis of paragangliomas that arise from the neural crest is considerably better than that of neuroendocrine tumors of epithelial origin (2).

Paragangliomas most often arise in the head and neck, specifically the carotid bifurcation and jugulo-tympanic region (1). However, paragangliomas of the larynx occur much less frequently. These slow-growing tumors emanate from either of the two paired paraganglia - the superior or inferior $(1,3)$. The superior paraganglia, located in the false vocal cord, are associated with the superior laryngeal nerve and artery. The inferior paraganglia, located near the lateral margin of the cricoid cartilage in the cricotracheal membrane, are associated with the recurrent laryngeal nerve $(1,3)$.

Laryngeal paragangliomas are more frequent in the supraglottis than in the subglottis and have a 3:1 female-to-male ratio (4). They tend to occur between the fourth and sixth decades of life. Symptoms depend on tumor location, but they most typically comprise dysphonia, dysphagia, stridor, or globus sensation.

We report a case of a patient diagnosed with laryngeal paraganglioma who presented with chronic cough as the primary complaint; to the best of our knowledge, this is the first case wherein chronic cough was the primary presenting symptom for this condition. 


\section{Case Presentation}

Consent for publication was obtained from the patient. The patient is a 77-year-old male who presented for the evaluation of a 3 -month history of chronic cough refractory to antibiotics and antiallergics. The patient denied symptoms of dysphagia additionally, he denied a smoking history. Flexible laryngoscopy revealed a large submucosal supraglottic mass emanating from the region of the right false vocal cord and right aryepiglottic fold (Figure 1). Movement of the right true vocal cord was decreased because of the presence of the mass, whereas that of the left true cord was normal. Postcontrast computed tomography (CT) scan of the neck showed a well-defined intensely enhancing mass arising from the right paraglottic space and bulging into the right pyriform sinus (Figure 2). The right aryepiglottic fold was medially displaced by the mass. Based on the imaging findings, diagnoses of paraganglioma and schwannoma were considered. Lymphadenopathy was absent.

Based on the laryngoscopic and CT findings, the most likely diagnosis was a benign process such as a paraganglioma, schwannoma, or granuloma. Therefore, it was decided to perform microsuspension laryngoscopy and attempt carbon dioxide $\left(\mathrm{CO}_{2}\right)$ laser resection for treating the patient. Following a mucosal incision, the mass was visualized and biopsies were taken. The procedure was aborted because of the high vascularity of the mass and significant bleeding with minimal manipulation. Final pathology was consistent with paraganglioma. The neoplastic cells were positive for synaptophysin, chromogranin, CD 56, and S-100 (Figures 3, 4) and negative for cytokeratin 8/18, A1/A3, CD3, CD20, desmin, and GFAP. This biomarker profile supported the final diagnosis of paraganglioma.

Because of the lateral and submucosal position of the tumor, the decision was made to perform a complete submucosal excision of the mass via a right transcervical approach with

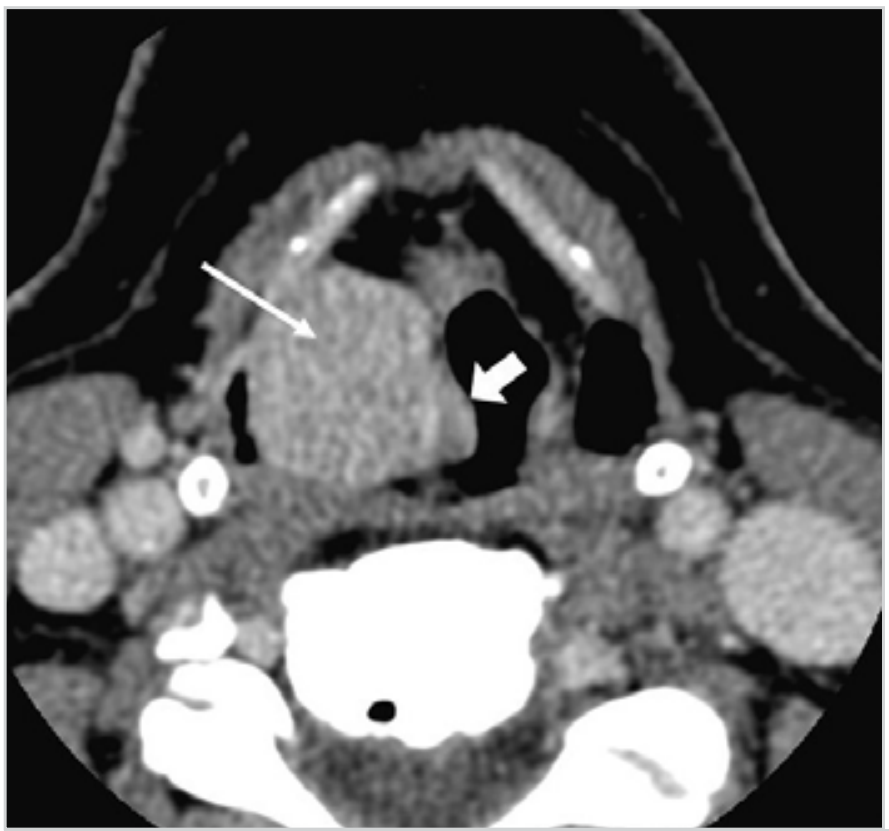

tracheostomy for airway protection. The anterior border of the sternocleidomastoid muscle was retracted laterally to gain access to the lateral component of the thyroid cartilage. The mass was palpable deep in the paraglottic space and extended into the right pyriform sinus. Therefore, inferior constrictors were dissected off the thyroid cartilage and retracted anteromedially for adequate visualization of the submucosal mass. Subsequently, the mass was partially dissected off the thyroid cartilage in a submucosal plane. A portion of the mass was adherent to the mucosa of the pyriform sinus and false vocal cord, necessitating resection of this mucosa. The specimen was $4.0 \mathrm{~cm}$ in diameter. The defect in the pyriform sinus and false vocal cord mucosa was meticulously closed, and a leak test was performed prior to closing the neck incision.

The postoperative course was uneventful, and the patient was discharged on postoperative day 7 , tolerating a soft diet. His

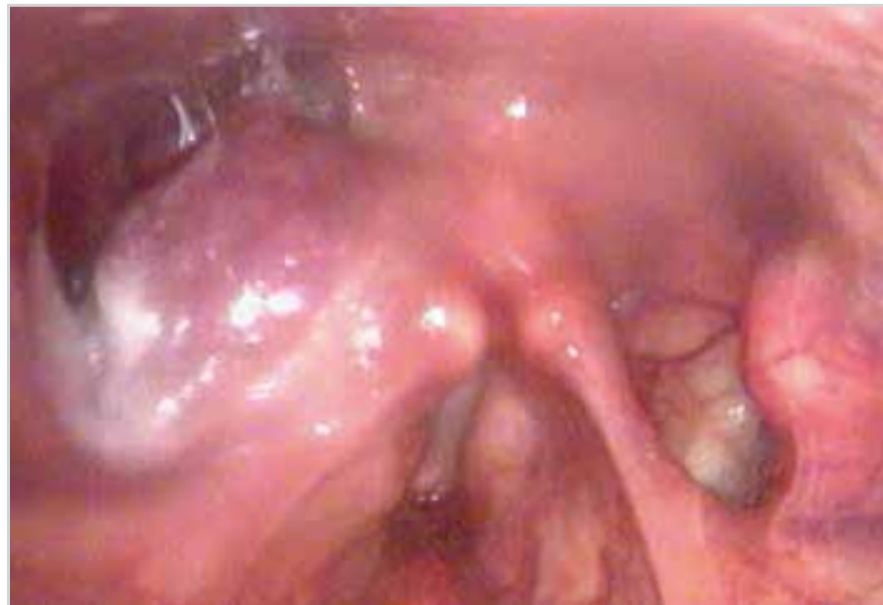

Figure 1. Preoperative flexible laryngoscopy showing right submucosal supraglottic mass

Figure 2. a, b. Postcontrast computed tomography scan (a: axial; b: coronal), revealing a well-defined intensely enhancing mass (thin arrow) that is arising from the right paraglottic space, bulging into the right pyriform sinus, and medially displacing the right aryepitlgottic fold (thick arrow) 


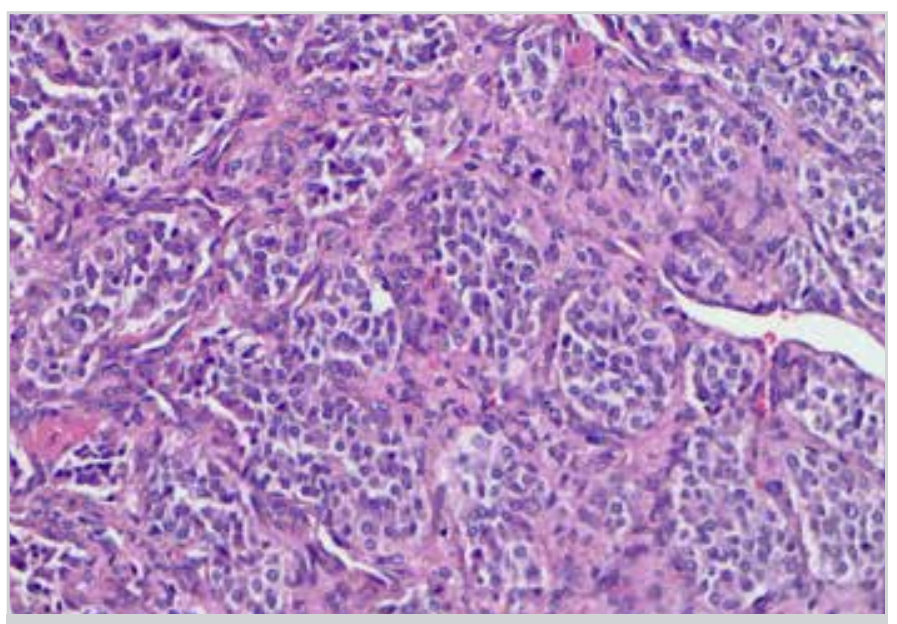

Figure 3. Hemotoxylin and eosin stain of tumor cells arranged in "Zellballen pattern" nests (X20 magnified)

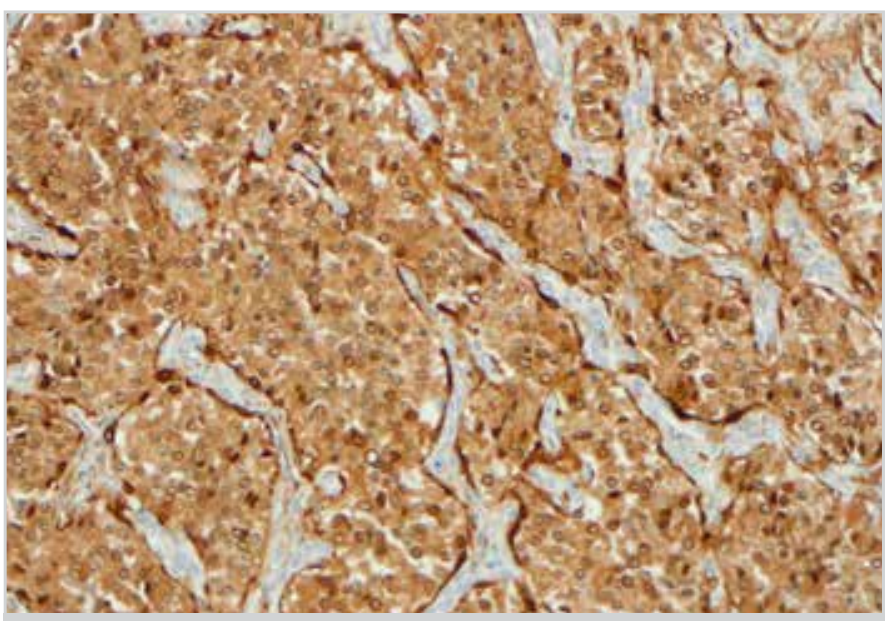

Figure 4. S-100 immunostain highlighting the sustentacular tumor cells (X20 magnified)

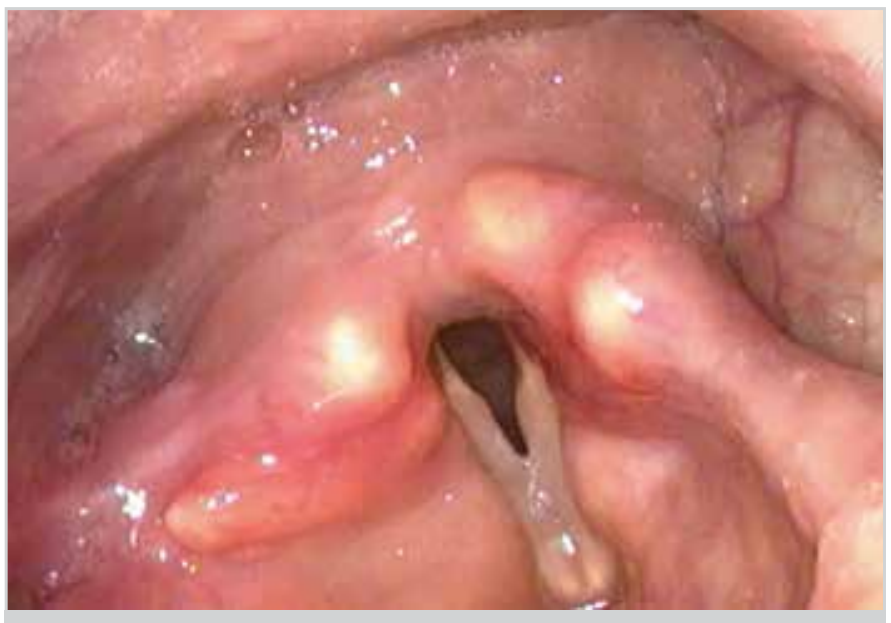

Figure 5. Postoperative flexible laryngoscopy showing resolution of the submucosal mass

tracheostomy was removed 1 month after surgery. Postoperative laryngoscopy performed at the time of decannulation showed resolution of the submucosal mass (Figure 5). On follow-up three months postoperatively, the patient had complete resolution of his chronic cough with return of normal movement of the right true vocal cord.

\section{Discussion}

Laryngeal paragangliomas are extremely rare neuroendocrine tumors of neural crest origin. They must be distinguished from epithelial-derived and other nonepithelial-derived neuroendocrine neoplasms arising near the larynx because of differences in prognosis and treatment $(1,2,5)$. Epithelial neuroendocrine tumors include carcinoid (typical and atypical), small-cell, and large-cell neuroendocrine carcinoma, whereas nonepithelial neuroendocrine tumors include variants of squamous cell carcinoma and medullary thyroid carcinoma (6). Immunohistochemistry is useful in making this distinction (1). The chief and sustentacular cells of paragangliomas are immunoreactive to neuroendocrine marker stains, such as chromogranin and synaptophysin, but not to epithelial markers, such as cytokeratin $(1,2,5)$.

Presenting symptoms associated with laryngeal paraganglioma typically include hoarseness due to vocal fold impingement, as well as dysphagia, dyspnea, or globus sensation for larger lesions (1). However, to the best of our knowledge, no case of chronic cough as the primary presenting symptom of laryngeal paraganglioma has been reported to date. Tissue for diagnosis is often obtained using microlaryngoscopy and biopsy. However, as observed in this case, the high vascularity and propensity to bleed can make this approach less desirable (4). Prior reports have noted that diagnostic biopsy could be avoided in these patients (1). Myssiorek et al. (1) have claimed that CT or magnetic resonance imaging (MRI), in combination with Indium-111-labeled pentetreotide scintigraphy, can be used in lieu of biopsy. In particular, triple-phase contrast-enhanced $\mathrm{CT}$ can detect the presence of a hypervascular lesion by showing intense contrast enhancement during inspiration, a peak level during Valsalva, and washing out during phonation (7). Characteristic MRI findings for paraganglioma include multiple serpentine and punctate areas of signal void within the tumor matrix, which represents the high-velocity flow of intramural blood vessels. Some authors have also supported the use of selective preoperative angiography to further aid in diagnosis, delineate the blood supply of the tumor, and allow for potential embolization of the tumor to decrease bleeding during operative excision $(1,4,8)$.

Treatment techniques include endoscopic removal, microlaryngoscopy with laser excision, and open resection via a transcervical, lateral thyrotomy, or laryngofissure approach. Endoscopic techniques are successful with smaller lesions but have been associated with higher rates of recurrence. Open techniques allow for dissection in the submucosal plane, thereby providing superior exposure and incurring less harm on swallowing (1). Shortterm tracheostomy is often performed for airway protection in the immediate postoperative period with early decannulation upon the resolution of postoperative edema (4). The open approach with temporary tracheostomy performed in the present case allowed for thorough excision, repair, and uncomplicated recovery.

\section{Conclusion}

Laryngeal paragangliomas are rare tumors that present with dysphonia, dysphagia, stridor, or globus sensation. This is the 
first case report of a patient presenting with chronic cough as the primary complaint. Imaging and nuclear radiology or histopathology with immunohistochemistry can establish the diagnosis. Prognosis is excellent with complete resection and a low risk of recurrence.

Informed Consent: Written informed consent was obtained from the patient who participated in this study.

Peer-review: Externally peer-reviewed.

Author Contributions: Concept - I.H., R.G., M.J., P.P., C.M., A.R.; Design - I.H., R.G., M.J., P.P., C.M., A.R.; Supervision - I.H., R.G., M.J., P.P., C.M., A.R.; Materials - I.H., R.G., M.J.; Data Collection and/or Processing - R.G., M.J., P.P.; Analysis and/or Interpretation I.H., R.G., M.J.; Literature Search - C.M., P.P., A.R.; Writing - P.P., A.R., C.M.; Critical Reviews - A.R., I.H., P.P., C.M., R.G., M.J.

Acknowledgements: The authors would like to thank all the faculty within the Departments of Otorhinolaryngology - Head and Neck Surgery, Pathology, and Radiology at Rush University Medical Center in Chicago, IL for their guidance and mentorship.

Conflict of Interest: The authors have no conflicts of interest to declare.
Financial Disclosure: The authors declared that this study has received no financial support.

\section{References}

1. Myssiorek D, Rinaldo A, Barnes L, Ferlito A. Laryngeal paraganglioma: an updated critical review. Acta Otolaryngol 2004; 124 : 995-9. [CrossRef]

2. Zhou X, Jiang S, Li H. A case of laryngeal paraganglioma and literature review. Int J Clin Exp Med 2015; 8: 16934-6.

3. Sharifkashany S, Yazdani N, Ghazavi H, Tajdini A. Laryngeal paraganglioma: a case report. Iran J Radiol 2014; 11: e21011. [CrossRef]

4. Kaytaz A, Karaman E, Edizer DT, Haciyev Y, Oz B. Laryngeal paraganglioma: report of an unusual entity. Ear Nose Throat J 2010; 89: 550-2.

5. Young WF Jr. Paragangliomas: clinical overview. Ann N Y Acad Sci 2006; 1073: 21-9. [CrossRef]

6. Hunt JL, Ferlito A, Hellquist H, Rinaldo A, Skálová A, Slootweg PJ, et al. Differential dagnosis in neuroendocrine neoplasms of the larynx. Adv Anat Pathol 2017; 24: 161-8. [CrossRef]

7. Çelebi İ, Bozkurt G, Mahmutoğlu AS. Paraganglioma of the larynx diagnosed with maneuvered three-phase contrast-enhanced computed tomography. Ear Nose Throat J 2018; 97: E10-3.

8. Del Gaudio JM, Muller S. Diagnosis and treatment of supraglottic laryngeal paraganglioma: report of a case. Head Neck 2004; 26 : 94-8. [CrossRef] 